\title{
A Novel Technique for Optimal Power Allocation Efficient Spectrum Sharing Radio Cognitive Networks
}

\author{
CH.V. Satwik Reddy, G. Siva Nageswara Rao*, Naresh Vurukonda and T. Gunasekhar \\ Department of Computer Science and Engineering, Koneru Lakshmaiah University, Green Fields, \\ Vaddeswaram, Guntur 522502, Andhra Pradesh, India; \\ vsatwikproject15@gmail.com, sivanags@gmail.com,naresh.vurukonda@gmail.com, tgunasekhar@kluniversity.in
}

\begin{abstract}
Cognitive Radio (CR) innovation helps in contriving remote framework for satisfactory sending of radio range with it's foresee procedure, self-adjustment and range allotment. Range assignment is a sufficient technique for ease the shortage of radio range issue by permitting unlicensed clients (optional clients) to agree with authorized clients (essential clients) under the situation of shielding the later from antagonistic obstruction. In this paper, we consideration on the throughput expansion of range designation Intellectual Radio (IR) systems and present an inventive subjective radio plan that will fundamentally propel their achievable throughput all the more accurately, a novel recipient and a pen structure for range portion is presented. The issue of fantastic force distribution approach that boosts the ergodic amount of the framework under moderate transmits and impedance power restriction are additionally considered.
\end{abstract}

Keywords: Cognitive Radio, Optimal Power Allocation, Spectrum Sharing, Throughput Maximization

\section{Introduction}

As remote correspondence procedures build up quickly, heterogeneous remote correspondence framework should be suited in characterized recurrence data transmission. Conventional orthogonal shocking detachment of heterogeneous framework is not sufficiently sufficient to sponsorship the persistent development of remote extra conveying condition, which makes range allotment among heterogeneous framework more basic. There are two noteworthy complaints for the eventual fate of remote plan: remote ranges appear and the high information rate utilization of expanding number of clients. IR is a novel disposition of telecom frameworks to unravel the predicament of range accessibility by restates underutilized authorized thickness groups. In a psychological radio framework, unlicensed remote clients (auxiliary clients) are stipend to get to the authorized groups progressively under the weight of adequate clash to the Primary Users (PU's). Range coating and underlay are the genuine systems which can assist the range designation among Secondary Users (SUs) and Pus ${ }^{-}$. In the coating approach, the SU imparts some portion of its energy advantages for the PU to organize a hand-off advantage transmission. Subsequently, the optional system make amends the interest obstruction by expanding the sign to interruption in addition to clamor proportion

Signal-to-Interference-plus-Noise Ratio (SINR) of essential acceptor. At that point the essential thought of coating methodology is to allocate power and channel

${ }^{*}$ Author for correspondence 
sponsorship to entire system, while utilize the prerequisites of PUs. As of late, to influence the otherworldly capacity loss of restricted half duplex, two-way telecast has been actualized ${ }^{1}$. In the underlay get to, the SU utilizes the same range at the same time with the PU while keeping up or bettering the transmission of the PU by utilize signal preparing and order ${ }^{\circ}$. Agreeable interchanges and systems administration is another new correspondence innovation paradigm that permits disseminated hopeless in a remote system to plan through some conveyed transmission or sign change over to perceive another type of space grouping to battle the negative assets of blurring channels ${ }^{20}$. Agreeable Communication (AC) can offer high direct amount and precision in a productive and ease route by gather a virtual receiving wire cluster among single-radio wire swell that helpfully profit their reception apparatuses.

Subjective Radio: CR will prompt a development in remote correspondence with convincing effects on innovation and in addition conformity of range use to influenced existing blockade. Psychological radio, considering Software-Defined Radio (SDR) tolerant innovation, is exhorted without precedent for ${ }^{7-9}$ to understand a moldable and productive acknowledgment of range. Subjective radio is an improvement of SR which again shows up from SDR. In this manner, IR is the circuitous stride from a pliable physical layer to an adaptable framework in general similar to reconfigurable radio. The term subjective radio is acquired from "comprehension". As indicated by Wikipedia ${ }^{10}$ perception is appoint to as o Mental procedures of a unique, with specific connection o Mental states, for example, convictions, desire and aims o Information change over including learning and mindfulness o Description of the showing up advancement of learning and approach inside of a gathering Resulting from this clarification, the subjective radio is a mindful correspondence plot that proficiently utilizes range as a part of an insightful way. It self-governing facilitates the use of range in order unused radio range on the premise of ready range use. The grouping of range as being undiscovered and the way it is utilized includes administration, as this range may be essentially doled out to an authorized correspondence framework. This optional utilization of range is alluded to as steep range sharing, which is transported in Section 3.2.2. To empower straight forwardness to the buyer, passionate radios give other than comprehension in radio capacity administration additionally perception in record and applications. The mental activities of a subjective radio set up on the cognizance circle from ${ }^{8}$ are delineating Figure 1 Cognition is outlined at the case of moldable radio range acknowledgment and the use of client inclinations. In watching the atmosphere, the psychological radio pick about its activity. An early on exchanging on might prompt a real activity, while regular movement suggests an assertion making taking into account gaining from thought history and the thought of the specific condition of the earth. The Federal Communications Commission (FCC) has break down in ${ }^{11}$ the accompanying (less progressive) appearance that subjective radios can join to engage a more sufficient and adaptable use of range: o Frequency Agility - The radio can change its performing recurrence to propel its utilization in adjusting to the earth.

- Dynamic Frequency Selection (DFS) - The radio detects signals from close-by transmitters to pick an ideal operation environment.

- Adaptive Modulation - The transmission qualities and waveforms can be reconfigured to adventure all open doors for the use of range

- Transmit Power Control (TPC) - The transmission force is adjusted to full power limits when important from one viewpoint and to lower levels then again to permit more prominent sharing of range.

- Location Awareness - The radio can decide its area and the area of different gadgets working in the same range to advance transmission parameters for expanding range re-use.

- Negotiated Use - The psychological radio might have calculations empowering the sharing of range as far as prearranged assertions between a licensee and an outsider or on an impromptu/constant basis.

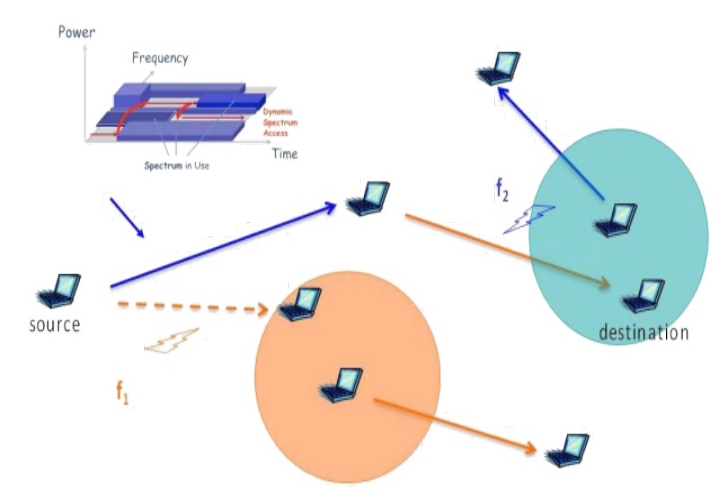

Figure 1. Radio cognitive network with spectrum. 


\section{Cognitive Radio}

CR is the empowering innovation for supporting element range gets to: the arrangement that addresses the range lack issue that is experienced in numerous nations. Consequently, CR is broadly viewed as a standout amongst the most encouraging advancements for future remote correspondences ${ }^{17}$. CR varies from ordinary radio gadgets in that a psychological radio can furnish clients with subjective ability and reconfigurability. Intellectual capacity alludes to the capacity to sense and assemble data from the encompassing environment, for example, data about transmission recurrence, transfer speed, power, tweak, and so on. With this ability, auxiliary clients can recognize the best accessible range. Reconfigurability alludes to the capacity to quickly adjust the operational parameters as indicated by the detected data so as to accomplish the ideal execution. By misusing the range in an artful manner, IR empowers optional clients to sense which partition of the range are accessible, select the best accessible channel, coordinate range access with different clients, and abandon the channel when an essential client recovers the range utilization right.

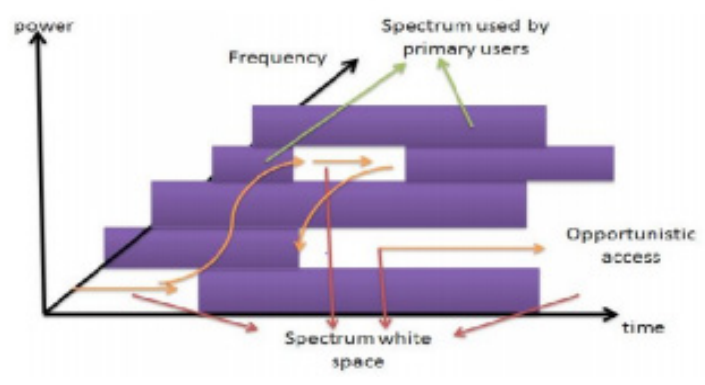

Figure 2. Spectrum white space.

Through range detecting and investigation, CR can identify the range white space (see Figure 1), i.e., a part of recurrence band that is not being utilized by the essential clients, and use the range. Then again, when essential clients begin utilizing the authorized range once more, CR can identify their movement through detecting, so that no destructive obstruction is created because of auxiliary clients' transmission. In this way range administration is done efficiently ${ }^{20}$. Since CRs can sense, distinguish, and screen the encompassing RF environment, for example, impedance and access accessibility, and reconfigure their own working attributes to best match outside circumstances, subjective correspondences can expand range effectiveness and backing higher data transfer capacity administration. In addition, the capacity of ongoing self-governing choices for effective range sharing likewise lessens the weights of incorporated range administration. Subsequently, CRs can be utilized in numerous applications. The fundamental elements of subjective radios are ${ }^{19}$ :

1. Range detecting

2. Power Control

3. Range administration

\subsection{Range Detecting}

Distinguishing unused range and sharing it, without destructive impedance to different clients; a vital prerequisite of the IR system to sense vacant range. Identifying essential clients is the most productive approach to distinguish void range. Range detecting systems might be gathered into three classes, for example, Transmitter recognition, Cooperative identification, and Interference-based discovery. Transmitter discovery Cognitive radios must have the capacity to figure out whether a sign from an essential transmitter is locally present in a specific range. There are a few proposed ways to deal with transmitter recognition to be specific Matched channel location, Energy Detection, CycloStationary component Detection. Helpful location Refers to range detecting techniques where data from different psychological radio clients is fused for essential client discovery.

\subsection{Power Control}

Power control is utilized for both sharp range access and range sharing CR frameworks for finding the cut-off level in SNR supporting the channel allotment and forcing impedance power imperatives for the essential client's security separately. In a joint force control and range detecting is proposed for limit expansion.

\subsection{Range Administration}

Catching the best accessible range to meet client correspondence necessities, while not making undue impedance to other (essential) clients. Psychological radios ought to settle on the best range band (of all groups accessible) to meet nature of administration necessities; consequently, range administration capacities are 
required for intellectual radios. Range administration capacities are delegated:

1. Spectrum investigation

2. Spectrum choice

3. Implementation:

In the subjective radio framework exhibited in Figure 1 let $g$ and $h$ (ergodic, stationary) denote the momentary channel power picks up from the auxiliary transmitter (SU-Tx) to the optional beneficiary (SU-Rx) and the essential recipient (PU-Rx), individually. In the accompanying, it is portrayed how the proposed range sharing plan works and present the collector and edge structure utilized in this psychological radio framework. By and by, the channel power pick up $h$ can be gotten through, e.g., evaluating the got signal force from the PU-Rx when it transmits, under the suppositions of the pre-learning on the PU-Rx transmit power level and the channel reciprocity.

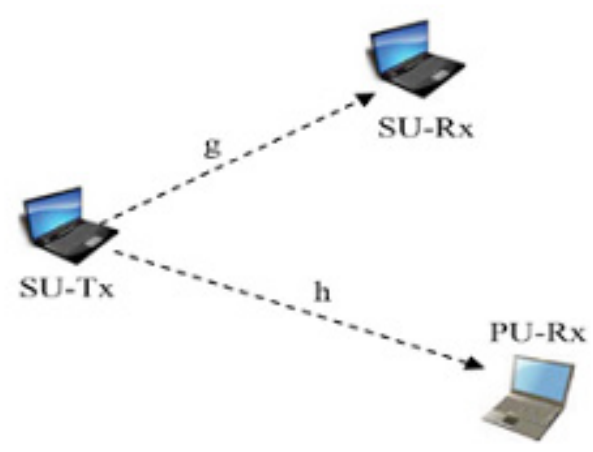

Figure 3. System model.

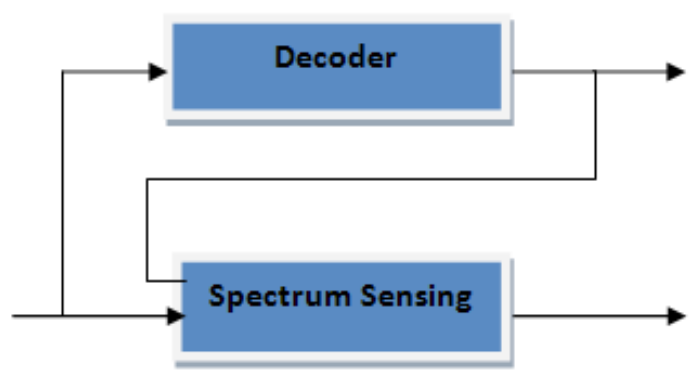

Figure 4. Receiver structure of the proposed CR system.

The beneficiary structure of the proposed IR framework is exhibited in Figure 2. They got motion at the auxiliary collector is given by

$$
y=\theta x_{p}+x_{s}+\mathrm{n}
$$

Where $\theta$ signifies the real status of the recurrence band $(\theta=1$ if the recurrence band is dynamic, though $\theta=0$ if the recurrence band is sit out of gear), $x p$ and xs speak to the got motion from the essential clients and the auxiliary transmitter, individually. At long last, $\mathrm{n}$ indicates the added substance commotion. They got flag y is at first gone through the decoder, where the flag from the auxiliary transmitter is acquired. In the accompanying, the flag from the optional transmitter is counterbalanced from the total got flag $y$ and the rest of the signal (2)

$$
y=\theta x_{p}+\mathrm{n}
$$

Is utilized to perform range detecting. Accordingly, rather than utilizing a constrained measure of time $t$ nearly the entire span of the casing $\mathrm{T}$ can be utilized for range detecting under the proposed psychological radio framework. Along these lines, we can perform range detecting and information transmission in the meantime and in this manner expand the length of both. In the beneficiary encourages the utilization of more mind boggling range detecting procedures that display expanded range detecting capacities, yet require higher detecting time, (for example, cyclostationary discovery, Generalized Likelihood Ratio Test based or covariancebased range detecting strategies), which precludes their application for brisk periodical range detecting under the edge structure.

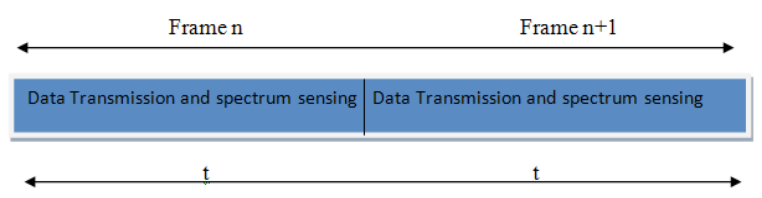

Figure 5. Frame structure of the proposed CR system.

The casing structure of the proposed subjective radio framework exhibited in above Figure both range detecting and information transmission are performed in the meantime utilizing the recipient structure as talked about before. The upside of the proposed outline structure is that the range detecting and information transmission times are at the same time augmented. The expanded detecting time empowers the location of extremely powerless signs from the essential clients in this manner decreasing false alert likelihood that would altogether uproot intermittence consequently expanding the throughput of the subjective radio system. Using the proposed outline structure prompts enhanced 
recognition likelihood, therefore better assurance of the essential clients from unsafe impedance which empowers a superior utilization of the accessible unused range. The false alert keeps the optional clients from getting to an unmoving recurrence band utilizing higher transmit power, and hence restrains their throughput according to prerequisite. The consistent range detecting can be accomplished under the proposed psychological radio framework, which guarantees better assurance of the essential systems.

\section{Ergodic Capacity of Proposed Spectrum Sharing Scheme}

In this area, the issue of determining the ideal force distribution system that expands the ergodic limit of the IR system that works under the proposed range sharing plan is talked about. In the proposed psychological radio framework, the optional clients adjust their transmit power toward the end of every edge in light of the choice of range detecting, and transmit utilizing higher force P0 when the recurrence band is distinguished to be sit without moving and lower force P1 when it is identified to be dynamic. Taking after the methodology of $\frac{6,12,17}{17}$, the immediate transmission rates when the recurrence band is idle $(\mathrm{H} 0)$ and dynamic $(\mathrm{H} 1)$ are given by

$$
\begin{aligned}
& r_{\downarrow} 0=\llbracket \log \rrbracket \downarrow_{\downarrow} 2 \llbracket\left(\mathbb{1} 1+\llbracket g p \rrbracket_{\downarrow} 0 /\left(\sigma_{\downarrow} \mathbf{n}^{\dagger} 2\right)\right) \\
& r_{1}=\log _{\downarrow} 2 \llbracket\left(\rrbracket_{1+} \llbracket g p \rrbracket_{\downarrow} 1 /\left(\sigma_{\downarrow} n^{\dagger} 2+\sigma_{\downarrow} p^{\uparrow} 2\right)\right)
\end{aligned}
$$

Individually, where $\sigma 2 p$ indicates they got power from the essential clients. The last parameter confines the achievable throughput of all range sharing psychological radio systems and shows the significance of range detecting and ideal force distribution on the throughput augmentation of range sharing IR systems. Be that as it may, the ideal range detecting may not be achievable practically speaking, where the real status of the essential clients may be dishonestly identified. In this manner, the four unique instances of momentary transmission rates in light of the real status of the essential clients (dynamic/ unmoving) and the choice of the auxiliary clients (essential client present/missing) as takes after:

$$
\begin{aligned}
& r_{\downarrow} 00=\llbracket \log \rrbracket_{\downarrow} 2 \mathbb{Q}\left(\mathbb{1}_{1+} \llbracket \operatorname{gp} \mathbb{1}_{\downarrow} 0 /\left(\sigma_{\downarrow} \mathrm{n}^{\top} 2\right)\right) \\
& r_{01}=\log _{\downarrow} 2\left(1+\frac{g p_{1}}{\sigma_{n}^{2}}\right) \\
& r_{10}=\log _{2}\left(1+\frac{g p_{0}}{\sigma_{n}^{2}+\sigma_{p}^{2}}\right)
\end{aligned}
$$

$$
r_{11}=\log _{\downarrow} 2 \llbracket\left(\mathbb{1}_{1+} \mathbb{\llbracket} g p \mathbb{1}_{\downarrow} \mathbf{1} /\left(\sigma_{\downarrow} n^{\dagger} \mathbf{2}+\sigma_{\downarrow} p^{\uparrow} \mathbf{2}\right)\right)
$$

Here, the main file number of the quick trans mission rates demonstrates the genuine status of the essential clients (" 0 " for idle, " 1 " for dynamic) and the second record number, the choice made by the auxiliary clients ("0" for truant, " 1 " for present) to keep the long haul control spending plan and adequately shield the essential clients from unsafe interference, We consider a normal (over all blurring states) transmit and impedance control requirement that can be detailed as takes after:

$$
\begin{aligned}
& E_{g . h}\left\{P\left(H_{0}\right)\left(1-P_{f a}\right) P_{0}+P\left(H_{0}\right) P_{f a} P_{1}+P\left(H_{1}\right)\left(1-P_{d}\right) P_{0}+P\left(H_{1}\right) P_{d} P_{1}\right\} \leq P_{a v} \\
& E_{g . h}\left\{P\left(H_{1}\right)\left(1-P_{d}\right) h P_{0}+P\left(H_{1}\right) P_{d} \boldsymbol{h} P_{1}\right\} \leq T
\end{aligned}
$$

Where $\mathrm{P}(\mathrm{H} 0)$ and $\mathrm{P}(\mathrm{H} 1)$ indicate the likelihood that the recurrence band is sit out of gear and dynamic, separately, Pd and Pfa speak to the recognition and false alert likelihood, individually, though Pav means the most extreme normal transmit force of the auxiliary clients, and $\Gamma$ the greatest normal impedance control that is mediocre by the essential clients. The purpose behind picking a normal impedance control requirement depends on the outcomes in $\frac{13,14}{}$, which demonstrate that a normal obstruction control limitation prompts to higher ergodic through put for the IR framework, and gives better security to the essential clients contrasted with a pinnacle impedance control imperative. At last, the advancement issue that expands the ergodic through put of the proposed range sharing psychological radio framework under joint normal transmit and obstruction control limitations can be planned as takes after Maximize

$$
\mathrm{C}=E_{g, k}\left\{P\left(H_{1}\right) P_{d} r_{11}+P\left(H_{0}\right) P_{f a} r_{01}+P\left(H_{1}\right)\left(1-P_{d}\right) r_{10}+P\left(H_{0}\right)\left(1-P_{f a}\right) r_{00}\right\}
$$

Subject to (4),(5),P0 $\geq 0, P \mathbf{1} \geq 0$. (6)

The Lagrangian with respect to the transmit powers $\mathrm{P} 0$ and

$\mathrm{P} 1$ is given by

$$
\mathrm{L}\left(P_{\mathbf{1}} \mathbf{0}, P_{\mathbf{1}}(\mathbf{1},) \boldsymbol{\lambda}, \mu\right)=
$$

Whereas the dual function can be obtained by

$$
\mathrm{D}(\boldsymbol{\lambda}, \mu)=\operatorname{supL}\left(P_{\downarrow} \mathbf{0}, P_{\downarrow} \mathbf{1}, \boldsymbol{\lambda}, \mu\right)
$$

Keeping in mind the end goal to ascertain the double capacity $d(\lambda, \mu)$, the supremum of the Lagrangian concerning the transmit powers P0 and P1 should be acquired. We accordingly apply the primal-dual decomposition technique $\frac{15,17}{}$, which encourage the 
arrangement of the joint advancement issue by decaying it into two raised single-variable enhancement issues, one for each of the transmit powers $\mathrm{P} 0$ and $\mathrm{P} 1$, as takes after:

Sub problem 1:

Maximize

$\{P 0 \geq 0\}$

$$
\begin{aligned}
& f_{\downarrow} 1\left(P_{\downarrow} \mathbf{0}\right)=E_{\downarrow}(g \cdot h)\left\{P ( H _ { \downarrow } 1 ) ( 1 - P _ { \downarrow } d ) \operatorname { l o g } _ { \downarrow } 2 \left(1+\llbracket g P \downarrow_{\downarrow} 0 /\right.\right. \\
& \left.\left(\sigma_{\downarrow} n^{\dagger} 2+\sigma_{\downarrow} p^{\dagger} 2\right)\right)+P\left(H_{1} 0\right)\left(1-P_{\downarrow} f a\right) \log _{\downarrow} 2(1+ \\
& \left.\left.\llbracket g P \rrbracket_{\downarrow} \mathbf{0} /\left(\sigma_{\downarrow} \eta^{\mathrm{T}} \mathbf{2}\right)\right)\right\}-\lambda E_{\downarrow}(g \cdot \boldsymbol{h})\left\{P\left(H_{\downarrow} \mathbf{0}\right)\left(\mathbf{1}-P_{\downarrow} f a\right) P_{\downarrow} 0\right. \\
& \left.+P\left(H_{\downarrow} \mathbf{1}\right)\left(\mathbf{1}-P_{\downarrow} d\right) P_{\downarrow} \mathbf{0}\right\}-\mu E_{\downarrow}(g \cdot h)\left\{P \left(H_{\downarrow} 1\right.\right.
\end{aligned}
$$

$\{P 1 \geq 0\}$

$f_{\mathbf{2}}=E_{g \cdot \mathbf{h}}\left\{P\left(H_{0}\right)\left(P_{f a}\right) \log _{\mathbf{2}}\left(1+\frac{g P_{1}}{\sigma_{n}^{2}}\right)+P\left(H_{1}\right)\left(P_{d}\right) \log _{2}\left(1+\frac{g P_{0}}{\sigma_{n}^{2}+\sigma_{\mathbf{p}}^{2}}\right)\right\}$

$-\lambda E_{g . h}\left\{P\left(H_{0}\right)\left(P_{f a}\right) P_{1}+P\left(H_{1}\right)\left(P_{d}\right) P_{1}\right\}-\mu E_{g . h}\left\{P\left(H_{1}\right)\left(P_{d}\right) h P_{1}\right\}(10)$

After forming their Lagrangian functions and applying the Karush-Kuhn-Tucker (KKT) conditions, the optimal powers $\mathrm{P} 0$ and $\mathrm{P} 1$ for given $\lambda, \mu$ are given by

$$
\begin{aligned}
& P_{0}=\left[\frac{A_{0}+\sqrt{\Delta_{0}}}{2}\right] \\
& P_{1}=\left[\frac{A_{1}+\sqrt{\Delta_{1}}}{2}\right]
\end{aligned}
$$

Where $[\mathrm{x}]+$ denotes $\max (0, \mathrm{x})$

$$
\begin{aligned}
& A_{0}=\frac{\log _{2}(e)\left(\alpha_{0}+\beta_{0}\right)}{\lambda\left(\alpha_{0}+\beta_{0}\right)+\mu \beta_{0} h}-\frac{2 \sigma_{n}^{2}+\sigma_{p}^{2}}{g} \\
& \boldsymbol{\Delta}_{0}=A_{0}^{2}-\frac{4}{g\left\{\frac{\sigma_{n}^{2}+\sigma_{p}^{2}}{g \sigma_{n}^{-2}}-\frac{\log _{2}(e)\left(\alpha_{0}\left(\sigma_{n}^{2}+\sigma_{p}^{2}\right)+\beta_{0} \sigma_{n}^{2}\right)}{\lambda\left(\alpha_{0}+\beta_{0}\right)+\mu \beta_{0} h}\right\}} \\
& A_{1}=\frac{\log _{2}(e)\left(\alpha_{1}+\beta_{1}\right)}{\lambda\left(\alpha_{1}+\beta_{1}\right)+\mu \beta_{1} h}-\frac{2 \sigma_{n}^{2}+\sigma_{p}^{2}}{g} \\
& \boldsymbol{\Delta}_{1}=A_{1}^{2}-\frac{4}{g\left\{\frac{\sigma_{n}^{2}+\sigma_{p}^{2}}{g \sigma_{n}^{-2}}-\frac{\log _{2}(e)\left(\alpha_{1}\left(\sigma_{n}^{2}+\sigma_{p}^{2}\right)+\beta_{1} \sigma_{n}^{2}\right)}{\lambda\left(\alpha_{1}+\beta_{1}\right)+\mu \beta_{1} h}\right\}}
\end{aligned}
$$

And the Parameters in the above equation are given by

$$
\begin{aligned}
& \alpha_{\mathbf{1}} \mathbf{0}=P\left(H_{\downarrow} 0\right)\left(1-P_{\downarrow} f a\right) \\
& \beta_{1} \mathbf{0}=P\left(H_{\downarrow} 1\right)\left(1-P_{\downarrow} d\right) \\
& \alpha_{\mathbf{1}} \mathbf{1}=P\left(H_{\downarrow} 0\right)\left(P_{\downarrow} f a\right) \\
& \beta_{1} \mathbf{1}=P\left(H_{\downarrow} 1\right)\left(P_{\downarrow} d\right)
\end{aligned}
$$

\section{Conclusion}

In this manner in this paper, throughput augmentation of range sharing IR systems is accentuated and an imaginative psychological radio framework is recommended that essentially enhances throughput all the more particularly , a novel recipient and a casing structure for range sharing is additionally being presented. The issue of ideal force designation technique that augments the ergodic limit of the framework under normal transmit and impedance power limitations is likewise concentrated on.

\section{References}

1. Federal Communication Commission. Spectrum policy task force report. Washington, DC; 2002 Nov. p. 02-155.

2. Mitola J, Maguire GQ. Cognitive radios: making software radio more personal. Institute of Electrical and Electronics Engineers (IEEE) Personal Communications. 1999 Aug; 6(4):13-8.

3. Ghasemi, Sousa ES. Fundamental limits of spectrum sharing in fading environments. Institute of Electrical and Electronics Engineers (IEEE) Transactions on Wireless Communications. 2007 Feb; 6(2):649-58.

4. Kang X, Liang YC, Garg HK, Zhang L. Sensing-based spectrum sharing in CR networks. Institute of Electrical and Electronics Engineers (IEEE) Transactions on Vehicular Technology. 2009 Oct; 58(8):4649-54.

5. Stotas S, Nallanathan A. Optimal sensing time and power allocation in multiband CR networks. Institute of Electrical and Electronics Engineers (IEEE) Transactions on Communications. 2011 Jan; 59(1):226-35.

6. Gunasekhar T, Rao KT, Reddy VK, Kiran PS, Rao BT. Mitigation of insider attacks through multicloud. International Journal of Electrical and Computer Engineering. 2015; 5(1):136.

7. Liang YC, Zeng Y, Peh ECY, Hoang AT. Sensing-throughput tradeoff for CR networks. Institute of Electrical and Electronics Engineers (IEEE) Transactions on Wireless Communications. 2008 Apr; 7(4):1326-37.

8. Musavian L, Aïssa S. Ergodic and outage capacities of spectrum sharing systems in fading channels. In the Proceedings of Institute of Electrical and Electronics Engineers (IEEE) Global Communications Conference (GLOBECOM), Washington, DC; 2007 Nov. p. 3327-31.

9. Kang X, Liang YC, Nallanathan A, Garg HK, Zhang R. Optimal power allocation for fading channels in CR networks: Ergodic capacity and outage capacity. Institute of Electrical and Electronics Engineers (IEEE) Transactions on Wireless Communications. 2009 Feb; 8(2):940-50. 
10. Gunasekhar T, Rao KT. Electronic Brake Control Module (EBCM): Single encryption, multiple decryptions. International Journal of Applied Engineering Research. 2014; 9(19):5885-93.

11. Srinivasa S, Jafar SA. CR networks: how much spectrum sharing is optimal? In the Proceedings of Institute of Electrical and Electronics Engineers (IEEE) Global Telecommunications Conference; 2007 Nov. p. 3149-53.

12. Wadhwa M, Xin C, Song M, Park EK. Throughput analysis for a contention -based dynamic spectrum sharing model. Institute of Electrical and Electronics Engineers (IEEE) Transaction on Wireless Communications. 2010 Apr; 9(4).

13. Gunasekhar T, Rao KT, Basu MT. Understanding insider attack problem and scope in cloud. In Circuit, Power and Computing Technologies (ICCPCT), International Conference on Institute of Electrical and Electronics Engineers (IEEE); 2015 Mar. p. 1-6.

14. Gunasekhar T, Rao KT, Basu MT. Understanding insider attack problem and scope in cloud. In Circuit, Power and Computing Technologies (ICCPCT), International Conference on on Institute of Electrical and Electronics Engineers (IEEE); 2015 Mar. p. 1-6.

15. Bhattacharjee $S$, Bhattacharjee $S$, Konar A, Roy $P$. Throughput analysis for a dynamic spectrum sharing model with finite PU's and infinite SUs. IEEE International Conference on Computer Science and Information Technology (ICCSIT); 2011 10-12 Jun. International Journal of Machine Learning and Computing. 2011 Oct; 1(4).

16. Vurukonda N, Rao BT, Reddy BT. A secured cloud data storage with access privileges. International Journal of Electrical and Computer Engineering (IJECE). 2016 Oct; 6(5):2338-44.

17. Rao BT. A study on data storage security issues in cloud computing. Procedia Computer Science. 2016; 92:128-35. 